TRANSACTIONS OF THE

AMERICAN MATHEMATICAL SOCIETY

Volume 363, Number 7, July 2011, Pages 3473-3488

S 0002-9947(2011)05224-4

Article electronically published on February 10, 2011

\title{
HARMONIC DIFFERENTIALS AND INFINITE GEODESIC JOINING TWO PUNCTURES ON A RIEMANN SURFACE
}

\author{
THÉRÈSE FALLIERO
}

\begin{abstract}
Let $M$ be a hyperbolic Riemann surface of finite volume. The harmonic dual form to an infinite geodesic joining two punctures on $M$ is obtained in two different ways. First of all, using the degeneration of hyperbolic Eisenstein series, it is made explicit in terms of these. Secondly, generalizing the construction of Kudla and Millson to the case of an infinite geodesic joining two punctures, we give an automorphic realization of this harmonic form.
\end{abstract}

\section{INTRODUCTION}

Many applications of degeneration of compact Riemann surfaces can be found in the literature: here is a nonexhaustive list dealing with, for example, the asymptotic behavior of Green's functions ([14, 11]), theta functions and the Riemann matrix (4]), Petersson series ([32]), Faltings invariant ([29]), eigenfunctions of the Laplacian and its eigenvalues ([10, 11, [14, 30, 31, 32, 33]), and Eisenstein series ([31], 22], 23], 24], 25]). In this paper, we answer in particular some questions in [16] (pp. 194 and 208) and in 6] (p. 562-09). In the first part we recall the definitions and results we need, and we also give the example of the principal congruence subgroup of level 2, $\Gamma(2)$ (see Proposition 1.1). In the second part we obtain the harmonic dual 1-form to an infinite geodesic joining two punctures by using the degeneration of hyperbolic Eisenstein series (see Proposition 2.3). Moreover, in the third part we answer a question that P. Gérardin asks in [6] (see Theorem 3.2): can we extend the Kudla and Millson construction to the case of $(p, q)$, with the geodesic joining the punctures $p$ and $q$ and orthogonal to the horocycles at $p$ and at $q$ ? In doing this we realize the representation of any cohomology class in $H^{1}(M, \mathbb{C})$ by a harmonic form exemplifying the ideas of Harder $(7,[8])$. We hope this work can give an automorphic realization of the modular symbol of Mazur (see Remark 3.1), and can be generalized to the higher dimensional case ([17, [18]) and in the case of infinite volume hyperbolic surfaces.

\section{Preliminary Definitions AND Results}

1.1. Definitions. In the following, $M$ will be a hyperbolic Riemann surface of finite volume; that is to say, $M$ has for universal covering space the upper half plane $\mathcal{H}$. The fundamental group of $M$ is identified to a Fuchsian group of the first

Received by the editors February 20, 2009.

2010 Mathematics Subject Classification. Primary 53C20, 53C22, 58A10; Secondary 58D27.

Key words and phrases. Harmonic differential, Eisenstein series.

(C)2011 American Mathematical Society 
kind, denoted by $\Gamma$. We'll suppose it has only two cusps on $M, p$ and $q$. It is easy to verify that all the results are true if there are cusps other than $p$ and $q$.

In [16, Kudla and Millson construct the harmonic 1-form dual to a simple closed geodesic on $M$. Let us recall the definition:

Definition 1.1. Let $\eta$ be a simple closed geodesic or an infinite geodesic joining $p$ and $q$. A 1-form $\alpha$ is dual to $\eta$ if for any closed 1-form with compact support $\omega$,

$$
\int_{M} \omega \wedge \alpha=\int_{\eta} \omega
$$

Equivalently, for any closed oriented cycle $c^{\prime}$, we have

$$
\int_{c^{\prime}} \alpha=\eta \cdot c^{\prime}
$$

(here $\eta \cdot c^{\prime}$ denotes the intersection number of the cycles $\eta$ and $c^{\prime}$ ).

We are interested in the 1-form dual to $(p, q)$ and which is harmonic. We begin by proving, in an elementary way, the uniqueness of the cohomology class and the existence of such a form. For this, recall the following results:

Lemma 1.1. Let $\alpha$ be a 1-form such that

(1) $\int_{M} \alpha \wedge \beta=0$ for each closed 1-form $\beta$ with compact support, or

(2) for any simple closed curve $c, \int_{c} \alpha=0$.

Then there exists a function $f$ with $\alpha=d f$.

Let us also recall the theorem in 28$]$.

Theorem 1.1 (Theorem 3, p. 106). Let $X$ be a compact Riemann surface. Let $p$ and $q$ be two distinct points on $X$ connected by a simple, oriented curve $L$, and let $X^{*}$ be the region obtained by cutting along $L$. There exists a unique differential of the first kind $d F(p, q)$ which is regular in $X$ apart from simple poles at $p$ and $q$, with residues 1 and -1 , respectively. The periods of $d F(p, q)$ on $X^{*}$ are all real, and for every loop which crosses $L$ exactly once from right to left, the imaginary part of the period is $2 \pi$.

Then the $1 / 2 \pi$ imaginary part of $d F(p, q)$ gives the dual form to the curve $L$.

The authors of [16] obtain the harmonic dual 1-form to a simple closed geodesic $\eta$ as the analytic continuation at $s=1$ of the hyperbolic Eisentein series, $\hat{\eta}^{s}$, associated to $\eta$. This hyperbolic Eisenstein series is defined by taking the sum over the set $H(\eta)$ of all the oriented geodesics $Y$ of $\mathcal{H}$ whose projection is $\eta$, of the 1-form:

$$
\omega_{Y}^{s}=\left\|\omega_{Y}\right\|^{s-1} \omega_{Y},
$$

where $\omega_{Y}=* P_{Y}^{*} V_{Y}, P_{Y}$ is the orthogonal projection on $Y, V_{Y}$ is the volume form of $Y$ and $\left\|\omega_{Y}\right\|$ denotes the pointwise norm of $\omega_{Y}$. The 1-form $\omega_{Y}^{s}$ is normalised so that the integral over any fiber of $P_{Y}$ is 1 :

$$
\int \frac{1}{k(s-1)} \omega_{Y}^{s}=1, k(s-1)=\frac{\Gamma(1 / 2) \Gamma(s / 2)}{\Gamma(1 / 2+s / 2)} .
$$


Then, for $\operatorname{Re} s>1$,

$$
\begin{aligned}
\hat{\eta}^{s} & =\frac{1}{k(s-1)} \sum_{Y \in H(\eta)} \omega_{Y}^{s} \\
& =\frac{\Gamma(1 / 2+s / 2)}{\Gamma(1 / 2) \Gamma(s / 2)} \sum_{\Gamma_{1} \backslash \Gamma} \gamma^{*} \frac{d x_{2}}{\left(\operatorname{ch} x_{2}\right)^{s}},
\end{aligned}
$$

where $\Gamma_{1}$ denotes the stabilizer group in $\Gamma$ of some geodesic $Y \in H(\eta)$ and any point $P \in \mathcal{H}$ is located by its Fermi coordinates $\left(x_{1}, x_{2}\right)$ associated to the geodesic $Y$.

Now recall the definition of the classical Eisentein series.

The stabilizer group of a cusp $\mathfrak{a}$ is an infinite cyclic group generated by a parabolic motion, say

$$
\Gamma_{\mathfrak{a}}=\{\gamma \in \Gamma: \gamma \mathfrak{a}=\mathfrak{a}\}=\left\langle\gamma_{\mathfrak{a}}\right\rangle .
$$

There exists $\sigma_{\mathfrak{a}} \in S L_{2}(\mathbb{R})$ such that $\sigma_{\mathfrak{a}} \infty=\mathfrak{a}, \sigma_{\mathfrak{a}}^{-1} \gamma_{\mathfrak{a}} \sigma_{\mathfrak{a}}=\left(\begin{array}{cc}1 & 1 \\ * & 1\end{array}\right)$. We call $\sigma_{\mathfrak{a}}$ a scaling matrix of the cusp $\mathfrak{a}$; it is determined up to composition with a translation from the right. The Eisenstein series for the cusp $\mathfrak{a}$ is then defined by

$$
E_{\mathfrak{a}}(z, s)=\sum_{\Gamma_{\mathfrak{a}} \backslash \Gamma} y\left(\sigma_{\mathfrak{a}}^{-1} \gamma z\right)^{s}
$$

where $s$ is a complex variable, $\operatorname{Re} s>1$.

An Eisenstein series of weight 2 associated to a cusp $\mathfrak{a}$ is the 1-form, defined for Re $s>1$, by

$$
\mathcal{E}_{\mathfrak{a}}(s, z)=\sum_{\gamma \in \Gamma_{\mathfrak{a}} \backslash \Gamma} y\left(\sigma_{\mathfrak{a}}^{-1} \gamma z\right)^{s-1} d\left(\sigma_{\mathfrak{a}}^{-1} \gamma z\right)=\frac{E_{\mathfrak{a}, 1}(s, z)}{y} d z,
$$

with

$$
E_{\mathfrak{a}, 1}(s, z)=y \sum_{\gamma \in \Gamma_{\mathfrak{a}} \backslash \Gamma} y\left(\sigma_{\mathfrak{a}}^{-1} \gamma z\right)^{s-1}\left(\left(\sigma_{\mathfrak{a}}^{-1} \gamma\right)^{\prime} z\right) .
$$

By definition, a standard horocyclic Eisenstein series, called for the sake of convenience a horocyclic Eisenstein series, is $\hat{\mathfrak{a}}^{s}(z)=\operatorname{Im}\left(\mathcal{E}_{\mathfrak{a}}(s, z)\right)$.

Remark 1.1. (1) In the notation of [5], $E_{\mathfrak{a}, 1}(s, z) \in \mathcal{F}_{1}$, where $\mathcal{F}_{1}$ is the space of all functions $f$ on $\mathcal{H}$ with $f(\gamma z)=\left(\frac{c z+d}{c \bar{z}+d}\right) f(z), \quad \gamma \in \Gamma$.

(2) A standard horocyclic Eisenstein series is associated to a horocycle of length 1: if $|\xi|$ is the length of the horocycle and $\hat{\xi}^{s}$ is the horocyclic Eisenstein series associated to $\xi$, then the 1 -form $|\xi|^{-s} \hat{\xi}^{s}$ depends only on the cusp (for more details see [6]).

1.2. Example. Now we are ready to treat the explicit example of the geodesic $(0, i \infty)$ on $M=\Gamma(2) \backslash \mathcal{H}$, where $\Gamma(2)$ is the principal congruence subgroup of level 2 :

$$
\Gamma(2)=\left\{\left(\begin{array}{ll}
a & b \\
c & d
\end{array}\right) \in S L_{2}(\mathbb{Z}), a=d=1 \quad \bmod 2, b=c=0 \quad \bmod 2\right\} .
$$

In this case we obtain (see [2])

$$
\mathcal{E}_{\infty}(1, z)-\mathcal{E}_{0}(1, z)=\theta(0, z)^{4} d z,
$$


where $\theta(0, z)^{4}=\left(\sum_{n \in \mathbb{Z}} e^{i \pi n^{2} z}\right)^{4}=\sum_{n \in \mathbb{N}} r_{4}(n) e^{i \pi n z}$ and $r_{4}(n)$ is the number of representations of $n$ as a sum of 4 squares. Let $c_{\infty}$ be the horocycle $\{y=1\}$ associated to the cusp $\infty\left(c_{\infty}\right.$ cross exactly once from right to left $\left.(i \infty, 0)\right)$. We easily verify that

$$
\begin{aligned}
\int_{c_{\infty}} \operatorname{Re}\left(\theta(0, z)^{4} d z\right) & =\int_{[-1 / 2,3 / 2]}\left(1+\sum_{n \geq 1} r_{4}(n) e^{-n \pi y_{o}} \cos n \pi x\right) d x \\
& =2
\end{aligned}
$$

and, with $\sigma: z \rightarrow-1 / z$ and $c_{0}=\sigma\left(c_{\infty}\right)$, the corresponding horocycle associated to the cusp 0:

$$
\int_{c_{0}} \operatorname{Re}\left(\theta(0, z)^{4} d z\right)=-2 .
$$

Moreover we have (with the notation of Remark 1.1) $\left|c_{\infty}\right|=\left|c_{0}\right|=2$, so we have the following propositions.

Proposition 1.1. The 1-form $-\operatorname{Re} \frac{1}{2}\left(\mathcal{E}_{\infty}(1, z)-\mathcal{E}_{0}(1, z)\right)=-\operatorname{Re}\left(\frac{1}{2} \theta(0, z)^{4} d z\right)$ is the harmonic dual form to $(0, i \infty)$.

Remark 1.2. (1) We recall that the space of a holomorphic differential on a finite volume Riemann surface of genus $g$ with $k \geq 0$ punctures, factored by the subspace of exact holomorphic differentials, is of dimension $2 g+k-1$. In the case of $\Gamma(2) \backslash \mathcal{H}$ a basis of this space is then $\mathcal{E}_{\infty}(1, z)-\mathcal{E}_{0}(1, z)$, $\mathcal{E}_{\infty}(1, z)-\mathcal{E}_{1}(1, z)$.

(2) We note that a square integrable holomorphic differential on a Riemann surface of finite volume corresponds to a cusp form of weight 2 (that is, a holomorphic form of weight 2 for $\Gamma$, with a vanishing zero Fourier coefficient in each of the cusps). Let $\mathcal{S}_{2}(\Gamma)$ denote such a space for $\Gamma \backslash \mathcal{H}$ of genus $g$. We know that it is of dimension $g$, and in the case of $\Gamma=\Gamma(2)$ we again verify that $\operatorname{dim} \mathcal{S}_{2}(\Gamma(2))=0$.

\section{THE HARMONIC DUAL FORM AND DEGENERATION}

The aim of this section is to find the harmonic dual form to an infinite geodesic joining two cusps on a Riemann surface by using the degeneration of the hyperbolic Eisenstein series. Consider a family of compact Riemann surfaces $\left(S_{l}\right)_{l>0}$ degenerating as $l \rightarrow 0$ to $S_{0}$, the geodesic $c_{l}$ on $S_{l}$ (of length $l$ ), nonhomologous to zero, being pinched to form a pair of cusps on $S_{0}$.

We note that $p$ and $q$ are the two cusps of $S_{0}$ arising from pinching $c_{l}: p$ is the limit of the right side of the $c_{l}$-collar and $q$ is the limit of the left side of the $c_{l}$-collar. Without loss of generality we can represent $p$ and $q$ by $\infty$ and 0 , respectively. We denote for simplicity $\hat{c}_{l}^{s}=\hat{l}^{s}$ and the complex 1-form of type $(1,0)$ associated:

$$
\theta_{l}(s, z)=1 / 2\left(i \hat{l}^{s}(z)-* \hat{l}^{s}(z)\right) \text {. }
$$

There exist homeomorphisms $f_{l}$ from $S_{l} \backslash c_{l}$ to $S_{0}$, with $f_{l}$ tending to an isometry in the $C^{k}$-norm on compact subsets of $S_{l} \backslash c_{l}$. We have the results (for the details see [3]):

Theorem 2.1. There exists $\beta>0$ such that for $s \in\{\operatorname{Re} s>1-\beta\}$ the family of 1 -forms $\frac{1}{l^{s}} \theta_{l}\left(s, f_{l}^{-1}().\right)$ converges uniformly on compact subsets of $S_{0}$ to 
$\frac{1}{2 k(s-1)}\left[\mathcal{E}_{\infty}(s,)-.\mathcal{E}_{0}(s,).\right]$, and so the family of 1 -forms $\frac{1}{l^{s}} \hat{l}^{s}\left(s, f_{l}^{-1}().\right)$ converges uniformly on compact subsets of $S_{0}$ to $\frac{1}{k(s-1)}\left[\hat{\infty}^{s}-\hat{0}^{s}\right]$.

We deduce in particular that the family of 1 -forms $\left(\frac{1}{l} \theta_{l}\left(1, f_{l}^{-1}(.)\right)\right)_{l>0}$ converges uniformly on compact subsets of $S_{0}$ to $\frac{1}{2 \pi}\left[\mathcal{E}_{\infty}(1,)-.\mathcal{E}_{0}(1,).\right]$.

As an application and using a classical result on the degeneration of the canonical period matrix, we obtain the asymptotic behavior of the periods of the holomorphic 1 -form $\theta_{l}(1, z)$. Suppose $S_{0}$ is of genus $g$ and let $\left\{b_{j}, c_{j}\right\}_{j=2}^{g+1}$ be a geodesic canonical homology basis of the complementary of the cuspidal regions: the intersection matrix defined by the cup product has the form $\left(\begin{array}{cc}0 & I \\ -I & 0\end{array}\right)$.

We set $\pi_{l}=f_{l}^{-1}$ and denote by $b_{i}(l)$ (resp. $c_{i}(l)$ ) the unique geodesic in the homology class of $\pi_{l}\left(b_{i}\right)$ (resp. $\pi_{l}\left(c_{i}\right)$ ) for $i=2, \ldots, g+1$. We complete this family with $b_{1}(l)$ and $c_{1}(l)=c_{l}$ in order to form a geodesic canonical homology basis on $S_{l}:\left\{b_{i}(l), c_{i}(l)\right\}_{i=1}^{g+1}$.

We then have the following two propositions (see for example [5], [19], 3]):

Proposition 2.1. For $A \in\left\{b_{i}, c_{i}, i=2, \ldots, g+1\right\}$

$$
\lim _{l \rightarrow 0} \int_{A(l)} \frac{\theta_{l}(1, z)}{l}=\frac{1}{2 \pi} \int_{A} \mathcal{E}_{\infty}(1, z)-\mathcal{E}_{0}(1, z) .
$$

Proposition 2.2. For $l \rightarrow 0$, $\int_{b_{1}(l)} \theta_{l}(1, z)=O(1)$ and $\int_{c_{l}} \theta_{l}(1, z) \sim \frac{l}{2 \pi}$.

On the other hand, at the end of [16] we are left with the question of the harmonic dual form to an infinite geodesic joining two cusps. To make this form explicit, we decompose the harmonic 1 -form $* \hat{l}$ on the following basis of harmonic differentials: $\left\{\hat{b}_{i}(l), i=1, \ldots, g+1, \quad \hat{c}_{i}(l), i=1, \ldots, g+1\right\}$, where $\hat{b}_{i}(l)$ is the harmonic dual form to $b_{i}(l)$ (resp. $\hat{c}_{i}(l)$ is the harmonic dual form to $c_{i}(l)$ ), so with the notation $\theta_{l}=\theta_{l}(1, z)=1 / 2(i \hat{l}-* \hat{l})$,

$$
* \hat{l}=\sum_{i=1}^{g+1} e_{i} \hat{c}_{i}(l)+\sum_{i=1}^{g+1} d_{i} \hat{b_{i}}(l)
$$

with

$d_{i}=\int_{c_{i}(l)} * \hat{l}=-2 \int_{c_{i}(l)} \theta_{l}, 1 \leq i \leq g+1$ and $e_{i}=\int_{b_{i}(l)}-* \hat{l}=2 \int_{b_{i}(l)} \theta_{l}, 2 \leq i \leq g+1$.

Let $\alpha$ be a closed 1-form on $S_{0}$ with compact support and consider the corresponding $\alpha_{l}$ on $S_{l}$, i.e. $\alpha_{l}\left(\pi_{l}(w)\right)=\alpha(w), w \in S_{0}$. The 1-form $\alpha_{l}$ is closed on $S_{l}$. Therefore

$$
\begin{aligned}
\int_{S_{l}} \alpha_{l} \wedge * \hat{l} & =\sum_{i=1}^{g+1} e_{i} \int_{S_{l}} \alpha_{l} \wedge \hat{c}_{i}(l)+\sum_{i=1}^{g+1} d_{i} \int_{S_{l}} \alpha_{l} \wedge \hat{b}_{i}(l) \\
& =\sum_{i=1}^{g+1} e_{i} \int_{c_{i}(l)} \alpha_{l}+\sum_{i=1}^{g+1} d_{i} \int_{b_{i}(l)} \alpha_{l}
\end{aligned}
$$

\footnotetext{
${ }^{1}$ Erratum in [3]: a mistake of sign occurred on p. 372, line 16.
} 
SO

$$
\int_{S_{l}} \alpha_{l} \wedge \frac{* \hat{l}}{l}=\frac{d_{1}}{l} \int_{b_{1}(l)} \alpha_{l}+\frac{e_{1}}{l} \int_{c_{1}(l)} \alpha_{l}+\sum_{i=2}^{g+1} \frac{e_{i}}{l} \int_{c_{i}(l)} \alpha_{l}+\sum_{i=2}^{g+1} \frac{d_{i}}{l} \int_{b_{i}(l)} \alpha_{l} .
$$

As $\alpha$ is of compact support, for $l$ sufficiently small $\int_{c_{1}(l)} \alpha_{l}=0$ it remains that

$$
\int_{S_{l}} \alpha_{l} \wedge \frac{* \hat{l}}{l}=\frac{d_{1}}{l} \int_{(p, q)} \alpha+\sum_{i=2}^{g+1} \frac{e_{i}}{l} \int_{c_{i}} \alpha+\sum_{i=2}^{g+1} \frac{d_{i}}{l} \int_{b_{i}} \alpha .
$$

Moreover, we have the following behaviors (Propositions 2.1 and 2.2) for $i=$ $2, \ldots, g+1$ :

$$
\lim _{l \rightarrow 0} \frac{d_{i}}{l}=-\frac{1}{\pi} \int_{c_{i}} \mathcal{E}_{\infty}-\mathcal{E}_{0}, \quad \lim _{l \rightarrow 0} \frac{e_{i}}{l}=\frac{1}{\pi} \int_{b_{i}} \mathcal{E}_{\infty}-\mathcal{E}_{0}
$$

and

$$
\lim _{l \rightarrow 0} \frac{d_{1}}{l}=-\frac{1}{\pi}
$$

If we let $l$ go to 0 in (2.1) with $(p, q)=(i \infty, 0)$, we obtain

$$
\begin{aligned}
\int_{S_{0}} \alpha \wedge\left(-\frac{1}{\pi} \operatorname{Re}\left[\mathcal{E}_{\infty}-\mathcal{E}_{0}\right]\right)= & -\frac{1}{\pi} \int_{(i \infty, 0)} \alpha \\
& +\sum_{i=2}^{g+1}\left(\frac{1}{\pi} \int_{b_{i}} \mathcal{E}_{\infty}-\mathcal{E}_{0} \int_{c_{i}} \alpha-\frac{1}{\pi} \int_{c_{i}} \mathcal{E}_{\infty}-\mathcal{E}_{0} \int_{b_{i}} \alpha\right) .
\end{aligned}
$$

Proposition 2.3. Setting

$$
\begin{aligned}
\Theta(z)=-\operatorname{Re}\left[\mathcal{E}_{\infty}(1, z)-\mathcal{E}_{0}(1, z)\right] & -\sum_{i=2}^{g+1} \int_{b_{i}}\left(\mathcal{E}_{\infty}(1, .)-\mathcal{E}_{0}(1, .)\right) \quad \hat{c_{i}}(z) \\
& +\sum_{i=2}^{g+1} \int_{c_{i}}\left(\mathcal{E}_{\infty}(1, .)-\mathcal{E}_{0}(1, .)\right) \quad \hat{b_{i}}(z),
\end{aligned}
$$

where for $i=2, \ldots, g+1, \hat{b}_{i}$ (resp. $\hat{c}_{i}$ ) is the harmonic dual form to $b_{i}$ (resp. $c_{i}$ ) on $S_{0}$, we have, for any closed 1-form with compact support on $S_{0}, \alpha$,

$$
\int_{S_{0}} \alpha \wedge \Theta=\int_{(0, i \infty)} \alpha
$$

so $\Theta$ is the dual harmonic form to $(0, i \infty)$.

Remark 2.1. Using the equivalent definition (1.1) of a dual form and by the same arguments we can see that for $A \in\left\{b_{i}, c_{i}, i=2, \ldots, g+1\right\}, \int_{A} \mathcal{E}_{\infty}(1, z)-\mathcal{E}_{0}(1, z) \in \mathbb{R}$, and with $c_{\infty}\left(\right.$ resp. $\left.c_{0}\right)$ a horocycle at $\infty$ (resp. 0$), \int_{c_{\infty}} \mathcal{E}_{\infty}(1, z)-\mathcal{E}_{0}(1, z)=1$ $\left(\operatorname{resp} \cdot \int_{c_{0}} \mathcal{E}_{\infty}(1, z)-\mathcal{E}_{0}(1, z)=-1\right)$.

\section{Automorphic REALization}

3.1. Fourier expansion of the Eisenstein series. We begin by recalling the Fourier expansion of the following Eisenstein series (see for example [12]).

Let $\mathfrak{a}, \mathfrak{b}$ be cusps of $\Gamma$ and $\sigma=\operatorname{Re} s>1$. We have, uniformly in $z \in \mathcal{H}$, the implied constant depending on $s$ and the group $\Gamma$,

$$
E_{\mathfrak{a}}\left(\sigma_{\mathfrak{b}} z, s\right)=\delta_{\mathfrak{a} \mathfrak{b}} y^{s}+\phi_{\mathfrak{a} \mathfrak{b}}(s) y^{1-s}+O\left(\left(1+y^{-\sigma}\right) e^{-2 \pi y}\right),
$$


where $\delta_{\mathfrak{a} \mathfrak{b}}=1$ if $\mathfrak{a}, \mathfrak{b}$ are equivalent. Otherwise it vanishes and $\phi_{\mathfrak{a} \mathfrak{b}}(s)$ is a function defined with Kloosterman sums and gamma factors.

3.2. Eisenstein series of weight 2. We'll need to investigate the Fourier expansion of $\mathcal{E}_{\mathfrak{a}}$ for $\mathfrak{a}=\infty$ and 0 to obtain:

Proposition 3.1. We have the following asymptotic behaviors:

(1) at $\mathfrak{a}=\infty$,

$$
\mathcal{E}_{\infty}(1, z)=\left(1+O(1) 1 / y+O\left(e^{-2 \pi y}\right)\right) d z \text { and } \mathcal{E}_{0}(1, z)=O(1 / y) d z,
$$

(2) at $\mathfrak{a}=0$,

$$
\mathcal{E}_{\infty}(1, z)=O(1 / y) d z \text { and } \mathcal{E}_{0}(1, z)=\left(\frac{1}{c_{0}^{2} z^{2}}+O(1 / y)\right) d z \text { for some }
$$
$c_{0} \neq 0$.

Proof. To obtain the Fourier expansion of $\mathcal{E}_{\infty}$ at $\mathfrak{a}=\infty$, we use the spectral decomposition formula (see for example [5])

$$
E_{\infty, 1}(z)=y^{s}+y^{1-s} \psi_{0}(s)+\sum_{m \neq 0} \psi_{m}(s) W_{\frac{m}{|m|}, s-1 / 2}(4 \pi|m| y) e^{2 \pi i m x},
$$

where $W_{\frac{m}{|m|}, s-1 / 2}$ are Whittaker functions and the Fourier coefficients are given by

$$
\begin{aligned}
& \psi_{n}(s)=-\pi^{s} \frac{|n|^{s-1}}{\Gamma\left(s+\frac{n}{|n|}\right)} \sum_{c>0} \frac{1}{c^{2 s}} S_{1}(0, n, c), n \neq 0, \\
& \psi_{0}(s)=-\pi \frac{4^{1-s} \Gamma(2 s-1)}{\Gamma(s-1) \Gamma(s+1)} \phi_{0}(s)
\end{aligned}
$$

with $S_{1}(0, n, c)$ Kloosterman sums and $\phi_{0}(s)=\sum_{c>0} \frac{1}{c^{2 s}} \sum_{0 \leq d<c} 1$ (for more details see [5]). Therefore, we have

$$
\mathcal{E}_{\infty}(s, z)=\left(y^{s-1}+y^{-s} \psi_{0}(s)+\sum_{m \neq 0} \psi_{m}(s) W_{\frac{m}{|m|}, s-1 / 2}(4 \pi|m| y) / y e^{2 \pi i m x}\right) d z .
$$

The analytic continuation at $s=1$ of $\psi_{n}(s)$ is well known (see for example [12]). Here we only point out that $s=1$ is a regular value for $\mathcal{E}_{\infty}(s, z)$. In fact, the residue of $\phi_{0}(s)$ at $s=1$ is given by $\lim _{s \rightarrow 1}(s-1) \phi_{0}(s)=1 /(\pi \operatorname{Vol}(M))$. As $(1 / \Gamma)(0)=0$,

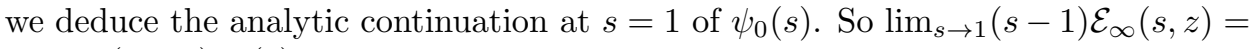
$\lim _{s \rightarrow 1}(s-1) \psi_{0}(s)=0$.

Moreover, we make use of the following asymptotic behavior to obtain the first equalities in the proposition: $W_{\mu, s-1 / 2}(t) \sim e^{-t / 2} t^{\mu}$, as $t \rightarrow+\infty$.

Now, the Fourier expansion of $\mathcal{E}_{\infty}$ at 0 is given in the form (see for example [12] p. 65) $\mathcal{E}_{\infty}\left(s, \sigma_{0} z\right)=\sum_{\tau \in \Gamma_{\infty} \backslash \Gamma \sigma_{0}} y(\tau z)^{s-1}\left(\tau^{\prime} z\right) d z$, where $\sigma_{0}$ is defined in the first paragraph. It is easy to see that we can choose $\sigma_{0}(z)=\frac{-1}{c_{0}^{2} z}$ and $\gamma_{0}(z)=\frac{z}{-c_{0}^{2} z+1}$ 
for some $c_{0} \neq 0$ :

$$
\begin{aligned}
\mathcal{E}_{\infty}\left(s, \sigma_{0} z\right) & =\sum_{d>0} \sum_{c} \sum_{n \in \mathbb{Z}} \frac{y^{s-1}(z)}{\left|d c_{0}(z+n)-c / c_{0}\right|^{2(s-1)}\left(d c_{0}(z+n)-c / c_{0}\right)^{2}} d z \\
& =\left(-y^{-s} \pi \frac{4^{s-1} \Gamma(2 s-1)}{\Gamma(s-1) \Gamma(s+1)} \sum_{d>0} \frac{1}{\left(d c_{0}\right)^{2 s}} \sum_{c\left(\bmod d c_{0}^{2}\right)} 1+O\left(e^{-2 \pi y}\right)\right) d z \\
& =\left(e^{s} y^{-s} \tilde{\psi}_{1}(s)+O\left(e^{-2 \pi y}\right)\right) d z .
\end{aligned}
$$

In a similar way we find the Fourier expansion of $\mathcal{E}_{0}$ at 0 and $\infty$ :

$$
\begin{aligned}
\mathcal{E}_{0}\left(s, \sigma_{0} z\right) & =\sum_{\tau \in \Gamma_{\infty} \backslash \sigma_{0}^{-1} \Gamma \sigma_{0}} y(\tau z)^{s-1}\left(\tau^{\prime} z\right) d z \\
& =\left(y^{s-1}+y^{-s} \sum_{b>0} \frac{1}{\left(-b c_{0}^{2}\right)^{2 s}} \sum_{a\left(\bmod b c_{0}^{2}\right)} 1+O\left(e^{-2 \pi y}\right)\right) d z .
\end{aligned}
$$

Now we remark that with the notation $\mathcal{E}_{\mathfrak{a}}(s, z, \Gamma)=\mathcal{E}_{\mathfrak{a}}(s, z)$,

$$
\mathcal{E}_{0}(s, z)=\mathcal{E}_{\infty}\left(s, \sigma_{0}^{-1} z, \sigma_{0}^{-1} \Gamma \sigma_{0}\right) .
$$

3.3. The Kudla and Millson construction of hyperbolic Eisenstein series. In [6], P. Gérardin asks the following question: if $\eta$ is the infinite geodesic $(p, q)$, can we do the same construction as Kudla and Millson, i.e., does the general term of the series (1.2) retain a meaning, does this series converge for $\operatorname{Re} s>1$, and does it define an analytic function in $s$ and admit an analytic continuation at $s=1$ which gives the harmonic dual form to $\eta$ ? Without loss of generality we suppose the two cusps $p$ and $q$ to be 0 and $\infty$, respectively, and, as the lift of the geodesic, we take the imaginary axis. As usual we suppose $\Gamma_{\infty}=\langle z \mapsto z+1\rangle$ to be the stabilizer of $\infty$ in $\Gamma$, and the stabilizer of $0, \Gamma_{0}$ is then generated by $z \mapsto \frac{z}{e z+1}$ (with the preceding notation $e=-c_{0}^{2}$ ). So the problem reduces to studying the following series:

$$
\hat{\eta}^{s}(z)=\frac{1}{k(s-1)} \sum_{\gamma \in \Gamma} \gamma *\left[\left(\frac{y}{|z|}\right)^{s-1} \operatorname{Im}\left(z^{-1} d z\right)\right]=\operatorname{Im}\left(\theta^{s}(z)\right),
$$

where

$$
\theta^{s}(z)=\frac{1}{k(s-1)} \sum_{\gamma \in \Gamma} \gamma *\left[\left(\frac{y}{|z|}\right)^{s-1} \frac{d z}{z}\right]
$$

and $k(s-1)=\frac{\Gamma(1 / 2) \Gamma(s / 2)}{\Gamma(1 / 2+s / 2)}$. Here is the answer to the first part of the question.

We have $\left\|\sum_{\gamma \in \Gamma} \gamma *\left(\frac{y}{|z|}\right)^{s-1} \operatorname{Im}\left(z^{-1} d z\right)\right\| \leq \sum_{\gamma \in \Gamma}\left(\frac{y}{|z|}\right)^{\sigma}(\gamma z)$, where $\sigma=\operatorname{Re} s$, and if we denote it by $\mathcal{S}=\sum_{\gamma \in \Gamma}\left(\frac{y}{|z|}\right)^{\sigma}(\gamma z)$, we have

$$
\mathcal{S}=\sum_{\gamma \in \Gamma_{\infty} \backslash \Gamma} y^{\sigma}(\gamma z) \sum_{n \in \mathbb{Z}} \frac{1}{|\gamma z+n|^{\sigma}} .
$$


Let $S_{z}$ be a system of representatives of $\Gamma_{\infty} \backslash \Gamma$ such that $|\operatorname{Re} \gamma z| \leq 1 / 2$; then

$$
\|\mathcal{S}\| \leq \sum_{\gamma \in S_{z}} \frac{y^{\sigma}(\gamma z)}{|\gamma z|^{\sigma}}+2 \sum_{\gamma \in S_{z}} y^{\sigma}(\gamma z) \sum_{n=1}^{\infty} \frac{1}{(n-1 / 2)^{\sigma}} .
$$

We first study $\sum_{\gamma \in S_{z}} \frac{y^{\sigma}(\gamma z)}{|\gamma z|^{\sigma}}$. We have

$$
\begin{aligned}
\sum_{\gamma \in S_{z}} \frac{y^{\sigma}(\gamma z)}{|\gamma z|^{\sigma}}= & \sum_{\Gamma_{0} \backslash S_{z}} \frac{y^{\sigma}(\gamma z)}{|\gamma z|^{\sigma}} \sum_{n \in \mathbb{Z}} \frac{1}{|n e \gamma z+1|^{\sigma}} \\
= & \sum_{\gamma \in \Gamma_{0} \backslash S_{z}} \frac{y^{\sigma}(\gamma z)}{|\gamma z|^{\sigma}} \\
& +\sum_{\gamma \in \Gamma_{0} \backslash S_{z}} \frac{y^{\sigma}(\gamma z)}{|\gamma z|^{\sigma}} \sum_{n \in \mathbb{Z}^{*}} \frac{1}{|n e|^{\sigma}\left[(x(\gamma z)+1 / n e)^{2}+y^{2}(\gamma z)\right]^{\sigma / 2}} .
\end{aligned}
$$

Let $K$ be a compact set in $\mathcal{H}$. We are going to prove that we can choose $m$ in $\mathcal{H}$ such that

$$
\forall z \in K, \forall \gamma \in \Gamma_{0} \backslash S_{z},|\gamma z| \geq|m| \text { and } \operatorname{Im} \gamma z \geq \operatorname{Im} m .
$$

Let's recall the following lemma; see also $([12$, p. 4):

Lemma 3.1. Let $\Gamma$ be a finite volume group which is not co-compact. Let a be a cusp of $\Gamma, z \in \mathcal{H}$ and $Y>0$. We have

$$
\#\left\{\gamma \in \Gamma_{a} \backslash \Gamma: \operatorname{Im} \sigma_{a}^{-1} \gamma z>Y\right\}<1+\frac{10}{c_{a} Y},
$$

where $c_{a}=\min \left\{c>0:\left(\begin{array}{cc}* & * \\ c & *\end{array}\right) \in \sigma_{a}^{-1} \Gamma \sigma_{a}\right\}$.

Now we have the following lemma.

Lemma 3.2. Let $K$ be a compact of $\mathcal{H}$. There exist a constant $C>0$ such that

$$
\forall z \in K, \forall \gamma \in \Gamma_{\infty} \backslash \Gamma, \operatorname{Im}(\gamma z) \leq C .
$$

Proof. We fix a compact $K \subset \mathcal{H}$, with $\delta$ its hyperbolic diameter. Let $z_{0} \in K$. We can suppose, without loss of generality, that $\left|\operatorname{Re} z_{0}\right| \leq 1 / 2$. We then verify

$$
\#\left\{\gamma \in \Gamma_{\infty} \backslash \Gamma: \operatorname{Im} \gamma z_{0}>\frac{10}{c_{\infty}} e^{\delta} \sqrt{1+\left(c_{\infty} / 20\right)^{2}}\right\} \leq 1 .
$$

We get into the most general situation with respect to the result of Lemma 3.2 .

$$
\#\left\{\gamma \in \Gamma_{\infty} \backslash \Gamma: \operatorname{Im} \gamma z_{0}>\frac{10}{c_{\infty}} e^{\delta} \sqrt{1+\left(c_{\infty} / 20\right)^{2}}\right\}=1 .
$$

Let $\gamma_{e x}$ be the unique element of $\Gamma_{\infty} \backslash \Gamma$ such that

$$
\operatorname{Im} \gamma_{e x} z_{0}>\frac{10}{c_{\infty}} e^{\delta} \sqrt{1+\left(c_{\infty} / 20\right)^{2}}>\frac{10}{c_{\infty}} .
$$

Then for all $\gamma \in \Gamma_{\infty} \backslash\left(\Gamma \backslash\left\{\gamma_{e x}\right\}\right)$ and for all $z \in K, \operatorname{Im} \gamma z \leq \frac{10}{c_{\infty}} e^{\delta} \sqrt{1+\left(c_{\infty} / 20\right)^{2}}$. To see this, let $\gamma \in \Gamma_{\infty} \backslash\left(\Gamma \backslash\left\{\gamma_{e x}\right\}\right)$ and $z \in K$; then $\operatorname{Im} \gamma z_{0} \leq 10 / c_{\infty}$. Moreover, with $d$ the hyperbolic distance and $p$ the orthogonal projection on the geodesic $(0, i \infty)$,

$$
d\left(p(\gamma z), p\left(\gamma z_{0}\right)\right) \leq d\left(\gamma z, \gamma z_{0}\right)=d\left(z, z_{0}\right) \leq \delta .
$$


Then

$$
\begin{aligned}
p(\gamma z) & \leq e^{\delta} p\left(\gamma z_{0}\right)=e^{\delta} \sqrt{\operatorname{Im}^{2} \gamma z_{0}+\operatorname{Re}^{2} \gamma z_{0}} \leq \frac{10}{c_{\infty}} e^{\delta} \sqrt{1+\left(c_{\infty} / 20\right)^{2}} \\
\operatorname{Im} \gamma z & \leq \frac{10}{c_{\infty}} e^{\delta} \sqrt{1+\left(c_{\infty} / 20\right)^{2}}
\end{aligned}
$$

Consequently, for all $\gamma \in \Gamma_{\infty} \backslash \Gamma$ and all $z \in K, \operatorname{Im} \gamma z \leq e^{\delta} \sqrt{\operatorname{Im}^{2} \gamma_{e x} z_{0}+1 / 4}$.

Now for $K$ a compact set of $\mathcal{H}$, let $c>0$ such that $\forall z \in K, \forall \gamma \in \Gamma_{0} \backslash \Gamma$, $\operatorname{Im} \sigma_{0}^{-1} \gamma z \leq c$. Let $m$ be the image by $\sigma_{0}$ of the intersection point of $\{\operatorname{Re} z=1 / 2\}$ and $\{\operatorname{Im} z=c\}$. We have

$$
\begin{aligned}
\sum_{\gamma \in S_{z}} \frac{y^{\sigma}(\gamma z)}{|\gamma z|^{\sigma}} & \leq \sum_{\gamma \in \Gamma_{0} \backslash S_{z}} \frac{y^{\sigma}(\gamma z)}{|m|^{\sigma}}+\sum_{\gamma \in \Gamma_{0} \backslash S_{z}} \frac{y^{\sigma}(\gamma z)}{|m|^{\sigma}} \sum_{n \in \mathbb{Z}^{*}} \frac{1}{|n e|^{\sigma}(\operatorname{Im} m)^{\sigma}} \\
& \leq \frac{1}{|m|^{\sigma}} \sum_{\Gamma_{\infty} \backslash \Gamma} y^{\sigma}(\gamma z)+2 \sum_{n \in \mathbb{N}^{*}} \frac{1}{(n e)^{\sigma}} \frac{1}{|m|^{\sigma}(\operatorname{Im} m)^{\sigma}} \sum_{\Gamma_{\infty} \backslash \Gamma} y^{\sigma}(\gamma z) .
\end{aligned}
$$

We deduce from the inequality

$$
\begin{aligned}
\|\mathcal{S}\| \leq & \frac{1}{|m|^{\sigma}} \sum_{\Gamma_{\infty} \backslash \Gamma} y^{\sigma}(\gamma z)+2 \sum_{n \in \mathbb{N}^{*}} \frac{1}{(n e)^{\sigma}} \frac{1}{|m|^{\sigma}(\operatorname{Im} m)^{\sigma}} \sum_{\Gamma_{\infty} \backslash \Gamma} y^{\sigma}(\gamma z) \\
& +2 \sum_{\Gamma_{\infty} \backslash \Gamma} y^{\sigma}(\gamma z) \sum_{n=1}^{\infty} \frac{1}{(n-1 / 2)^{\sigma}}
\end{aligned}
$$

the uniform convergence on all compact sets of $\mathcal{H}$ and all compact sets of $\operatorname{Re} s>1$. To conclude, we have the following theorem.

Theorem 3.1. For $\operatorname{Re} s>1$, the Eisenstein series associated to the geodesic $\eta=$ $(p, q)$ converges uniformly on all compact sets. It represents a $\mathcal{C}^{\infty}$ closed form which is dual to $\eta$. For $\operatorname{Re} s>1$ it satisfies the differential functional equation

$$
\Delta \hat{\eta}^{s}=s(1-s)\left[\hat{\eta}^{s}-\hat{\eta}^{s+2}\right] .
$$

Now we want to investigate the analytic continuation of $\hat{\eta}^{s}$ at $s=1$. For this, first of all, we are going to show that $\theta^{s}(z)+i\left(\mathcal{E}_{\infty}(1, z)-\mathcal{E}_{0}(1, z)\right)$ is square integrable. What we have to do is to investigate the Fourier expansion of $\theta^{s}$ at each inequivalent cusp 0 and $\infty$. We have the following proposition:

Proposition 3.2. At $\infty$

$$
\theta^{s}(z)=\left(\frac{1}{i}+O(1 / y)\right) d z
$$

and at 0

$$
\theta^{s}(z)=\left(-\frac{1}{i e z^{2}}+O(1 / y)\right) d z
$$

Proof. We begin by estimating the constant term of the Fourier expansion of $\theta^{s}$ at the cusp $\infty$. We write for $\sigma=\operatorname{Re} s>1$

$$
\theta^{s}(z)=\frac{1}{k(s-1)} \sum_{\gamma \in \Gamma_{\infty} \backslash \Gamma} \frac{y^{s-1}}{|c z+d|^{2(s-1)}(c z+d)^{2}} \zeta(\gamma z, s) d z
$$


where

$$
\zeta(Z, s)=\sum_{n \in \mathbb{Z}} \frac{1}{|n+Z|^{s-1}(n+Z)} .
$$

We have, where we denote by $\mathrm{I}_{\infty}$ the orbit of the identity modulo $\Gamma_{\infty}$,

$$
\begin{aligned}
\theta^{s}(z) & =\frac{1}{k(s-1)}\left[y^{s-1} \zeta(z, s)+\sum_{\gamma \in \Gamma_{\infty} \backslash \Gamma-\mathrm{I}_{\infty}} \frac{y^{s-1}}{\left.|c z+d|^{2(s-1)(c z+d)^{2}} \zeta(\gamma z, s)\right] d z}\right. \\
& =\frac{1}{k(s-1)}\left[y^{s-1} \zeta(z, s)+\sum_{S_{z}-\mathrm{I}_{\infty}} \frac{y^{s-1}}{|c z+d|^{2(s-1)}(c z+d)^{2}} \zeta^{*}(\gamma z, s)\right. \\
& \left.+\sum_{S_{z}-\mathrm{I}_{\infty}} \frac{y^{s-1}}{|c z+d|^{2(s-1)}(c z+d)^{2}} \frac{1}{|\gamma z|^{s-1}(\gamma z)}\right] d z .
\end{aligned}
$$

Here we introduced $S_{z}$ as a set of orbit representatives modulo $\Gamma_{\infty}$ such that

$$
|\operatorname{Re} \gamma z| \leq 1 / 2 \text { and } \zeta^{*}(Z, s)=\sum_{n \in \mathbb{Z}^{*}} \frac{1}{|n+Z|^{s-1}(n+Z)} .
$$

In order to study the Fourier expansion of $\zeta(z, s)$ at $\infty$, we observe that Poisson's summation formula is applicable here with the result

$$
\zeta(z, s)=\sum_{n=-\infty}^{+\infty} A_{n}(z, s), \operatorname{Im} z>0
$$

with

So we have

$$
A_{n}(z, s)=\int_{-\infty}^{+\infty} \frac{e^{-2 \pi i n v}}{(v+z)|v+z|^{s-1}} d v
$$

$$
\begin{aligned}
A_{0}(z, s) & =\int_{-\infty}^{+\infty} \frac{1}{(v+z)|v+z|^{s-1}} d v \\
& =\frac{1}{y^{s-1}} \int_{-\infty}^{+\infty} \frac{1}{(i+t)|i+t|^{s-1}} d t=\frac{1}{i y^{s-1}} \int_{-\pi / 2}^{+\pi / 2} \cos ^{s-1} \theta d \theta \\
& =\frac{k(s-1)}{i y^{s-1}} .
\end{aligned}
$$

Moreover, utilizing [27], p. 127, there exists a constant $C$, independent of $s$ and $n$, such that

$$
\left|A_{n}(z, t)\right| \leq C \frac{e^{-\pi|n| y}}{y^{\sigma}} .
$$

Therefore

Lemma 3.3. Near $\infty, \zeta$ has the following asymptotic behavior:

$$
\zeta(z, s)=\frac{k(s-1)}{i y^{s-1}}+O\left(\frac{e^{-\pi y}}{y^{s}}\right) .
$$

Return to the first term of $\theta^{s}$ in the sum $\frac{1}{k(s-1)} y^{s-1} \zeta(z, s) d z$. It can be written as

$$
\frac{1}{k(s-1)} y^{s-1} \zeta(z, s) d z=\left[\frac{1}{i}+O\left(\frac{e^{-\pi y}}{y^{s}}\right)\right] d z \text {. }
$$


Let us work on the investigation of the second term. For $\gamma \in S_{z}-\mathrm{I}_{\infty}$ we have

$$
\left|\sum_{n \in \mathbb{Z}^{*}} \frac{1}{|n+\gamma z|^{s-1}(n+\gamma z)}\right| \leq \sum_{n \in \mathbb{Z}^{*}} \frac{1}{|| n|-1 / 2|^{\sigma}}=2 \sum_{n \geq 1} \frac{1}{(n-1 / 2)^{\sigma}},
$$

and so

$$
\left|\sum_{S_{z}-\mathrm{I}_{\infty}} \frac{y^{s-1}}{|c z+d|^{2(s-1)}(c z+d)^{2}} \zeta^{*}(\gamma z, s)\right| \leq\left(\sum_{S_{z}-\mathrm{I}_{\infty}} \frac{y^{\sigma-1}}{|c z+d|^{2 \sigma}}\right) 2\left(2^{\sigma}-1\right) \zeta(\sigma),
$$

with $\sum_{n \geq 1}(n-1 / 2)^{-s}=\left(2^{s}-1\right) \zeta(s)$. To estimate this sum we use Subsection 3.1]

$$
\sum_{\Gamma_{\infty} \backslash \Gamma-\mathrm{I}_{\infty}} \frac{y^{\sigma}}{|c z+d|^{2 \sigma}}=O\left(y^{1-\sigma}\right) .
$$

Rewrite the last term in $\theta^{s}$ as follows and denote $S_{z}^{*}=S_{z}-\mathrm{I}_{\infty}$ :

$$
\begin{aligned}
& \sum_{S_{z}-\mathrm{I}_{\infty}} \frac{y^{s-1}}{|c z+d|^{2(s-1)}(c z+d)^{2}} \frac{1}{|\gamma z|^{s-1}(\gamma z)} \\
& \quad=\sum_{S_{z}-\mathrm{I}_{\infty}} \frac{y^{s-1}}{|c z+d|^{(s-1)}(c z+d)|a z+b|^{s-1}(a z+b)} .
\end{aligned}
$$

We have the inequalities:

$$
\begin{aligned}
& \left|\sum_{S_{z}-\mathrm{I}_{\infty}} \frac{y^{s-1}}{|c z+d|^{(s-1)}(c z+d)|a z+b|^{s-1}(a z+b)}\right| \\
& \quad \leq \sum_{\Gamma_{0} \backslash S_{z}^{*}} \frac{y^{s-1}}{|c z+d|^{2 \sigma}} \frac{1}{|\gamma z|^{\sigma}} \sum_{n \in \mathbb{Z}} \frac{1}{|n e \gamma z+1|^{\sigma}} \\
& \quad \leq \sum_{\Gamma_{0} \backslash S_{z}^{*}} \frac{y^{\sigma-1}}{|c z+d|^{2 \sigma}} \frac{1}{|\gamma z|^{\sigma}}+\sum_{\Gamma_{0} \backslash \Gamma} \frac{y^{\sigma-1}}{|c z+d|^{2 \sigma}} \frac{1}{|\gamma z|^{\sigma}} \sum_{n \in \mathbb{Z}^{*}} \frac{1}{|n e \gamma z+1|^{\sigma}} .
\end{aligned}
$$

First it follows that

$$
\begin{aligned}
\sum_{\Gamma_{0} \backslash S_{z}^{*}} \frac{y^{\sigma-1}}{|c z+d|^{2 \sigma}} \frac{1}{|\gamma z|^{\sigma}} & =\sum_{\Gamma_{0} \backslash S_{z}^{*}} \frac{y^{\sigma-1}}{|c z+d|^{\sigma}|a z+b|^{\sigma}} \\
& \leq\left(\sum_{\Gamma_{0} \backslash S_{z}^{*}} \frac{y^{\sigma-1}}{|c z+d|^{2 \sigma}}\right)^{1 / 2}\left(\sum_{\Gamma_{0} \backslash S_{z}^{*}} \frac{y^{\sigma-1}}{|a z+b|^{2 \sigma}}\right)^{1 / 2} .
\end{aligned}
$$

Now we have an estimation (see (1.1))

$$
\sum_{\Gamma_{0} \backslash S_{z}^{*}} \frac{y^{\sigma-1}}{|c z+d|^{2 \sigma}} \leq \sum_{\Gamma_{\infty} \backslash \Gamma-\mathrm{I}_{\infty}} \frac{y^{\sigma-1}}{|c z+d|^{2 \sigma}} .
$$

Moreover,

$$
\sum_{\Gamma_{0} \backslash S_{z}^{*}} \frac{y^{\sigma-1}}{|a z+b|^{2 \sigma}} \leq \sum_{\Gamma_{0} \backslash \Gamma} \frac{y^{\sigma-1}}{|a z+b|^{2 \sigma}},
$$

and we know the right term is an $O(1 / y)$ near $\infty$. 
The second sum to estimate is

$$
\sum_{\Gamma_{0} \backslash \Gamma} \frac{y^{\sigma-1}}{|c z+d|^{2 \sigma}} \frac{1}{|\gamma z|^{\sigma}} \sum_{n \in \mathbb{Z}^{*}} \frac{1}{|n e \gamma z+1|^{\sigma}}=\sum_{\Gamma_{0} \backslash \Gamma} \frac{y^{\sigma-1}}{|a z+b|^{2 \sigma} e^{\sigma}} \sum_{n \in \mathbb{Z}^{*}} \frac{1}{\left|n-\sigma_{0} \gamma z\right|^{\sigma}}
$$

with $\sigma_{0}(z)=-\frac{1}{e z}$. We can find a set of orbit representatives modulo $\Gamma_{0}$ such that $\left|\operatorname{Re}\left(-\sigma_{0} \gamma z\right)\right| \leq 1 / 2$, so the second term is majored by

$$
\sum_{\Gamma_{0} \backslash \Gamma} \frac{y^{\sigma-1}}{|a z+b|^{2 \sigma}} e^{\sigma} 2\left(2^{\sigma}-1\right) \zeta(\sigma)
$$

Then we investigate the Fourier expansion at the cusp 0. The method is similar, and we don't give all the details. We write

$$
\begin{aligned}
\theta^{s}(z) & =\frac{1}{k(s-1)} \sum_{\Gamma_{0} \backslash \Gamma} \frac{y^{s-1}}{|a z+b|^{2(s-1)}(a z+b)^{2} e^{s}} \zeta\left(-\sigma_{0} \gamma z, s\right) d z \\
& =\frac{1}{k(s-1)}\left(\frac{y^{s-1}}{|z|^{2(s-1)} z^{2} e^{s}} \zeta\left(-\sigma_{0} z, s\right) d z\right. \\
& \left.+\sum_{\Gamma_{0} \backslash \Gamma-\mathrm{I}_{0}} \frac{y^{s-1}}{|a z+b|^{2(s-1)}(a z+b)^{2} e^{s}} \zeta\left(-\sigma_{0} \gamma z, s\right) d z\right) .
\end{aligned}
$$

We easily compute the constant term of the Fourier expansion at 0 of $\zeta\left(-\sigma_{0} z, s\right)$ :

$$
\zeta\left(-\sigma_{0} z, s\right)=-\left(\frac{k(s-1)}{i\left(\frac{y}{e|z|^{2}} s-1\right.}\right)+O\left(e^{-\pi \frac{y}{e|z|^{2}}}\right) .
$$

Now we have the following estimates for the terms in the sum of $\theta^{s}$ :

$$
\begin{aligned}
& \left|\sum_{\Gamma_{0} \backslash \Gamma-\mathrm{I}_{0}} \frac{y^{s-1}}{|a z+b|^{2(s-1)}(a z+b)^{2} e^{s}} \zeta^{*}\left(-\sigma_{0} \gamma z, s\right)\right| \\
& \quad \leq \sum_{\Gamma_{0} \backslash \Gamma-\mathrm{I}_{0}} \frac{y^{\sigma-1}}{|a z+b|^{2 \sigma}} 2 \sum_{n \geq 1} \frac{1}{(n-1 / 2)^{\sigma}}, \\
& \quad \sum_{\Gamma_{0} \backslash \Gamma-\mathrm{I}_{0}} \frac{y^{s-1}}{|a z+b|^{2(s-1)}(a z+b)^{2} e^{s}} \frac{1}{\left(-\sigma_{0} \gamma z\right)\left|\sigma_{0} \gamma z\right|^{s-1} \mid} \\
& \quad \leq \sum_{\Gamma_{\infty} \backslash\left(\Gamma_{0} \backslash \Gamma-\mathrm{I}_{0}\right)} \frac{y^{\sigma-1}}{|c z+d|^{\sigma}|a z+b|^{\sigma}} \\
& \quad+\sum_{\Gamma_{\infty} \backslash\left(\Gamma_{0} \backslash \Gamma-\mathrm{I}_{0}\right)} \frac{y^{\sigma-1}}{|c z+d|^{2 \sigma}} \sum_{n \in \mathbb{Z}^{*}} \frac{1}{|n+\gamma z|^{\sigma}} .
\end{aligned}
$$

The second term of the right hand side is majored by

$$
\sum_{\Gamma_{\infty} \backslash \Gamma} \frac{y^{\sigma-1}}{|c z+d|^{2 \sigma}} 2 \sum_{n \geq 1} \frac{1}{(n-1 / 2)^{\sigma}}
$$


and the first term by

$$
\left(\sum_{\Gamma_{\infty} \backslash \Gamma} \frac{y^{\sigma-1}}{|c z+d|^{2 \sigma}}\right)^{1 / 2}\left(\sum_{\Gamma_{0} \backslash \Gamma-\mathrm{I}_{0}} \frac{y^{\sigma-1}}{|a z+b|^{2 \sigma}}\right)^{1 / 2} .
$$

By Propositions 3.1 and 3.2 we obtain the following proposition.

Proposition 3.3. The 1-forms

$$
\theta^{s}(z)-1 / i\left(\mathcal{E}_{\infty}(1, z)-\mathcal{E}_{0}(1, z)\right), \hat{\eta}^{s}(z)+\operatorname{Re}\left(\mathcal{E}_{\infty}(1, z)-\mathcal{E}_{0}(1, z)\right)
$$

are square integrable.

Denote by $\Phi^{s}(z)=\hat{\eta}^{s}(z)+\operatorname{Re}\left(\mathcal{E}_{\infty}(1, z)-\mathcal{E}_{0}(1, z)\right)$, and by the results in Proposition 3.3 and Theorem 3.1 that $\Phi^{s}$ is square integrable on $M$ and satisfies

$$
\Delta \Phi^{s}=s(1-s)\left[\Phi^{s}-\Phi^{s+2}\right] .
$$

Since $\Phi^{s}$ is square integrable on $M$, we can use the method developed in [16]. The 1 -form $\Phi^{s}$ may be expanded in eigenforms of $\Delta$ :

$$
\Phi^{s}(z)=\sum_{n=1}^{\infty} a_{n}(s) \nu_{n}(z)+\sum_{\mathfrak{a} \in\{0, \infty\}} \frac{1}{4 \pi i} \int_{-\infty}^{+\infty} h_{\mathfrak{a}}(s, t) \mathcal{E}_{\mathfrak{a}}(1 / 2+i t, z) d t,
$$

where $a_{n}(s)=\left\langle\Phi^{s}, \nu_{n}\right\rangle, h_{\mathfrak{a}}(s, t)=\left\langle\Phi^{s}, \mathcal{E}_{\mathfrak{a}}(1 / 2+i t,).\right\rangle$.

Now $h_{-\mathfrak{a}}(s, t)=\overline{h_{\mathfrak{a}}(s, t)}$ and $\Delta \nu_{n}=\lambda_{n} \nu_{n}$. By (3.2), $\Delta \Phi^{s}$ is square integrable and

$$
\begin{aligned}
\left\langle\Delta \Phi^{s}, \nu_{n}\right\rangle & =\left\langle\Phi^{s}, \Delta \nu_{n}\right\rangle=\lambda_{n}\left\langle\Phi^{s}, \nu_{n}\right\rangle \\
\left\langle\Delta \Phi^{s}, \mathcal{E}_{\mathfrak{a}}(1 / 2+i t, .)\right\rangle & =\left\langle\Phi^{s}, \Delta \mathcal{E}_{\mathfrak{a}}(1 / 2+i t, .)\right\rangle=\left(1 / 4+t^{2}\right)\left\langle\Phi^{s}, \mathcal{E}_{\mathfrak{a}}(1 / 2+i t, .)\right\rangle .
\end{aligned}
$$

So, by (3.2) we find the induction relations

(1) $a_{n}(s)=\frac{s(s-1)}{\lambda_{n}+s(s-1)} a_{n}(s+2)$

(2) $h_{\mathfrak{a}}(s, t)=\frac{s(s-1)}{1 / 4+t^{2}+s(s-1)} h_{\mathfrak{a}}(s+2, t)$.

From this we deduce, in particular, that $\Phi^{s}$ has a meromorphic continuation to $\operatorname{Re} s>1 / 2$ and $s=1$ is a regular value. Therefore we have the following theorem.

Theorem 3.2. The 1-form $\hat{\eta}^{s}$ has a meromorphic continuation to $\operatorname{Re} s>1 / 2$ with $s=1$ a regular point and $\hat{\eta}$ the harmonic dual form to $\eta$.

Remark 3.1. The Hecke congruence group of level $N, \Gamma_{0}(N)$, has no elliptic points. In this case we obtain an automorphic realization of the modular symbol of Mazur ([20]). For example, for $N=4$, let $\overline{\mathcal{H}}=\mathcal{H} \cup \mathbb{Q} \cup\{i \infty\}, Y=\Gamma_{0}(4) \backslash \overline{\mathcal{H}}=\Gamma(2) \backslash \overline{\mathcal{H}}$. Noting $h_{2}=\left(\begin{array}{cc}1 & 0 \\ 0 & 1 / 2\end{array}\right)$, we have $h_{2} \Gamma_{0}(4) h_{2}^{-1}=\Gamma(2)$ and, if for every $\gamma$ in $\Gamma(2), f(\gamma z)=f(z)$, then setting $g(Z)=f\left(h_{2} Z\right)$, the function $g$ verifies for every $\gamma$ in $\Gamma_{0}(4), g(\gamma Z)=g(Z)$. Consider the linear functional on differential forms:

$$
\{i \infty\}=\int_{(0, i \infty)}: H^{0}\left(Y, \Omega_{Y / \mathbb{C}}^{1}\right) \rightarrow \mathbb{C} .
$$


The modular symbol associated to $i \infty,\{i \infty\}$, is represented by

$$
\begin{aligned}
-\operatorname{Re} \frac{1}{2}\left(\mathcal{E}_{\infty}(1, z)-\mathcal{E}_{0}(1, z)\right) & =-\operatorname{Re} \frac{1}{2}\left(\theta(0, z)^{4} d z\right) \\
& =-\operatorname{Re} \lim _{s \rightarrow 1}\left(\theta^{s}(z)\right),
\end{aligned}
$$

where the 1 -form

$$
\begin{gathered}
\theta^{s}(z)=\left(\sum_{\substack{(m, n, p, q) \in \mathbb{Z}^{4} \\
(1+2 m)(1+2 q)-4 n p=1}} \frac{1}{|(2 m+1) z+2 n|^{s-1}|2 p z+(2 q+1)|^{s-1}}\right. \\
\left.\cdot \frac{d z}{((2 m+1) z+2 n)(2 p z+(2 q+1))}\right) .
\end{gathered}
$$

\section{REFERENCES}

1. L. V. Ahlfors and L. Sario, Riemann surfaces, Princeton Univ. Press., Princeton, 1960. MR 0114911 (22:5729)

2. T. Falliero, Décomposition spectrale de 1-formes différentielles sur une surface de Riemann et séries d'Eisenstein, Math. Ann., 317 (2000), 263-284. MR1764237(2001e:11055)

3. T. Falliero, Dégénérescence de séries d'Eisenstein hyperboliques, Math. Ann. 339 (2007), no. 2, 341-375. MR 2324723(2008m:32041)

4. J. Fay, Theta Functions on Riemann Surfaces, Lecture Notes in Math. 352, Springer-Verlag Edition (1973). MR0335789 (49:569)

5. J. Fay, Fourier coefficients of the resolvent for a Fuchsian group, J. Reine Angew. Math. 293-294 (1977), 144-203. MR0506038 (58:21944)

6. P. Gérardin, Formes automorphes associées aux cycles géodésiques des surfaces de Riemann hyperboliques, Séminaire Bourbaki 562 (1980).

7. G. Harder, On the cohomology of $S L_{2}(\theta)$, Lie groups and their representations. Proc. of the Summer School on Group Representations, ed. Gel'fand Hilger, London (1975), 139-150. MR.0425019 (54:12977)

8. G. Harder, On the cohomology of discrete arithmetically defined groups, Proc. of the Int. Coll. on Discrete Subgroups of Lie Groups and Applications to Moduli, Bombay, 1973. Oxford University Press (1975), 129-160. MR0425018 (54:12976)

9. D. A. Hejhal, Theta functions, kernel functions, and Abelian integrals, Mem. Amer. Math. Soc., 129, American Mathematical Society, Providence, R.I. (1972). MR0372187 (51:8403)

10. D. A. Hejhal, A continuity method for spectral theory on Fuchsian groups, Modular Forms, Chichester, Ellis-Horwood Edition (1984). MR803365 (87g:11063)

11. D. A. Hejhal, Regular b-groups, Degenerating Riemann surfaces and Spectral Theory, Mem. Amer. Math. Soc., 88 no. 437 (1990). MR1052555 (92h:11043)

12. H. Iwaniec, Introduction to the Spectral Theory of Automorphic Forms, Bibl. Rev. Mat. Iberoamericana, 1995. MR1325466 (96f:11078)

13. L. Ji, Spectral degeneration of hyperbolic Riemann surfaces, J. Differential Geom. 38 (1993), 263-313. MR1237486 (94j:58172)

14. L. Ji, The asymptotic behavior of Green's functions for degenerating hyperbolic surfaces, Math. Z. 212 (1993), 375-394. MR1207299 (94d:58152)

15. J. Jorgenson, Asymptotic behavior of Falting's delta function, Duke Math. J. 61 (1990), no. 1, 221-254. MR1068387 (91m:14042)

16. S. Kudla and J. Millson, Harmonic differentials and closed geodesics on a Riemann surface, Invent. Math. 54 (1979), 193-211. MR553218 (81a:53041)

17. S. Kudla and J. Millson, The Poincaré dual of a geodesic algebraic curve in a quotient of the 2-ball, Canad. J. Math. 33, vol. 2 (1979), 485-499. MR617638 (82f:32043)

18. S. Kudla and J. Millson, Geodesic cyclics and the Weil representation. I. Quotients of hyperbolic space and Siegel modular forms, Compos. Math. 45, vol. 2 (1982), 207-271. MR651982 (83m:10037) 
19. A. Lebowitz, On the degeneration of Riemann surfaces. Advances in the theory of Riemann surfaces, Ann. of Math. Stud. 66 (1971), 265-286. MR0281908(43:7622)

20. B. Mazur, Courbes elliptiques et symboles modulaires, Séminaire Bourbaki 414 (1972). MR 0429921 (55:2930)

21. D. Mumford, Tata lectures on theta. I, Progr. Math. 28 (1983). MR688651 (85h:14026)

22. K. Obitsu, Non-completeness of Zograf-Takhtajan's Kähler metric for Teichmüller space of punctured Riemann surfaces, Comm. Math. Phys. 205 (1999), 405-420. MR.1712579 (2001b:32026)

23. K. Obitsu, The asymptotic behavior of Eisenstein series and a comparison of the WeilPetersson and the Zograf-Takhtajan metrics, Publ. Res. Inst. Math. Sci. 37 (2001), 459-478. MR 1855431 (2002f:11116)

24. K. Obitsu and S. A. Wolpert, Grafting hyperbolic metrics and Eisenstein series, Math. Ann., 341 (2008), no. 3, 685-706. MR2399166 (2009d:32011)

25. K. Obitsu, Asymptotics of degenerating Eisenstein series. Arxiv:0801.3691(2008).

26. R. Phillips and P. Sarnak, On cusp forms for co-finite subgroups of $P S L(2, \mathbb{R})$, Invent. Math. 80 (1985), 339-364. MR.788414 (86m:11037)

27. H. Rademacher, Topics in Analytic Number Theory, Springer-Verlag, Berlin, Heidelberg 169 (1973). MR0364103 (51:358)

28. C. L. Siegel, Topics in complex function theory, Tracts in Mathematics Number 25, vol. 2, Wiley-Interscience, 1971.

29. R. Wentworth, The asymptotics of the Arakelov-Green's function and Faltings'delta invariant, Comm. Math. Phys. 137 (1991), 427-459. MR1105425 (92g:14019)

30. S. A. Wolpert, Asymptotics of the spectrum and the Selberg Zeta function on the space of Riemann surfaces, Comm. Math. Phys. 112 (1987), 283-315. MR905169 (89c:58136)

31. S. A. Wolpert, Spectral limits for hyperbolic surfaces. I, Invent. Math. 108 (1992), 67-89. MR:1156387 (93b:58160)

32. S. A. Wolpert, Spectral limits for hyperbolic surfaces. II, Invent. Math. 108 (1992), 91-129. MR.1156387 (93b:58160)

33. S. A. Wolpert, Disappearance of cusp forms in special families, Ann. of Math. 139 no. 2 (1994), 239-291. MR:1274093 (95e:11062)

LABORATOIRE D'ANALYSE NON LinÉAire ET GÉOMÉTRIE (E-A 251), Université D'Avignon et des Pays de Vaucluse, F-84018 Avignon, France

E-mail address: therese.falliero@univ-avignon.fr 